\title{
Microwave temperature and pressure measurements with the Odin satellite: I. Observational method
}

\author{
J.R. Pardo, M. Ridal, D. Murtagh, and J. Cernicharo
}

\begin{abstract}
The Odin satellite is equipped with millimetre and sub-millimetre receivers for observations of several molecular lines in the middle and upper atmosphere of our planet ( $\sim 25-100 \mathrm{~km}$, the particular altitude range depending on the species) for studies in dynamics, chemistry, and energy transfer in these regions. The same receivers are also used to observe molecules in outer space, this being the astrophysical share of the project. Among the atmospheric lines that can be observed, we find two corresponding to molecular oxygen $(118.75 \mathrm{GHz}$ and $487.25 \mathrm{GHz})$. These lines can be used for retrievals of the atmospheric temperature vertical profile. In this paper, we describe the radiative-transfer modeling for $\mathrm{O}_{2}$ in the middle and upper atmosphere that we will use as a basis for the retrieval algorithms. Two different observation modes have been planned for Odin, the three-channel operational mode and a high-resolution mode. The first one will determine the temperature and pressure on an operational basis using the oxygen line at $118.75 \mathrm{GHz}$, while the latter can be used for measurements of both $\mathrm{O}_{2}$ lines, during a small fraction of the total available time for aeronomy, aimed at checking the particular details of the radiative transfer near $\mathrm{O}_{2}$ lines at very high altitudes $(>70 \mathrm{~km})$. The Odin temperature measurements are expected to cover the altitude range $\sim 30-90 \mathrm{~km}$.
\end{abstract}

PACS Nos.: 07.57Mj, 94.10Dy, 95.75Rs

Résumé : Le satellite Odin est équipé de détecteurs millimétriques et sous-millimétriques pour observer plusieurs raies moléculaires dans les parties moyenne et supérieure de l'atmosphère (25-100 km, le domaine en altitude dépendant des molécules étudiées) afin d'étudier la dynamique, la chimie et les transferts d'énergie dans ces régions. Ces mêmes détecteurs peuvent être orientés vers l'espace dans le volet astrophysique de la mission. Parmi

Received 13 May 1999. Accepted 23 November 2001. Published on the NRC Research Press Web site at http://cjp.nrc.ca/ on 30 April 2002.

J.R. Pardo. ${ }^{1,2}$ LERMA, Observatoire de Paris-Meudon, 61, avenue de l'Observatoire, 75014 Paris, France; NASA-Goddard Institute for Space Studies, 2880 Broadway, New York, NY 10025, U.S.A.; and George W. Downs Laboratory of Physics, Mail Stop 320-47, Office 423, California Institute of Technology, 1200 E California Blvd., Pasadena, CA 91125, U.S.A.

M. Ridal and D. Murtagh. Department of Meteorology, Stockholm University, Svante Arrhenius v. 12, S-10691 Stockholm, Sweden.

J. Cernicharo. Consejo Superior de Investigaciones Científicas, IEM, Serrano 121, E-28006, Madrid, Spain.

${ }^{1}$ Present Address: Consejo Superior de Investigaciones Científicas, IEM, Serrano 121, E-28006, Madrid, Spain.

${ }^{2}$ Corresponding author (email: pardo@submm.caltech.edu). 
les raies atmosphériques observables se trouvent deux raies de l'oxygène à $118,75 \mathrm{GHz}$ et à 487,25 GHz. Ces raies permettent de déduire le profil vertical de la température. Nous présentons ici une modélisation du transfert radiatif pour $\mathrm{O}_{2}$ dans les parties moyenne et supérieure de l'atmosphère, utilisable comme base pour l'algorithme d'inversion de cette information. Odin est prévu pour deux modes d'opération, un mode à trois canaux et un mode de haute résolution. Le premier détermine régulièrement la pression et la température en utilisant la raie à $118,75 \mathrm{GHz}$, alors que l'autre mesure les deux raies pendant une petite partie du temps total consacré à l'étude de l'atmosphère, afin de vérifier certains détails du transfert radiatif dans le voisinage des raies d' $\mathrm{O}_{2}$ à très haute altitude $(>70 \mathrm{~km})$. Les mesures en température fournies par Odin doivent couvrir le domaine de 30 à 90 km d'altitude.

[Traduit par la Rédaction]

\section{Introduction}

The thermal emission from a gas in the atmosphere is a function of the local temperature and pressure and the amount of the emitting gas present (Sect. 2). A priori knowledge of the temperature and pressure makes it possible to derive the mixing ratio of the emitting gas. The retrievals often rely upon temperature and pressure $(T / P)$ information from climatological databases. To improve the retrievals of the measured constituents, prior determination of temperature and pressure using absorption bands of other molecules is often helpful. An analysis of the influence of temperature uncertainties on the retrievals of different atmospheric molecules that can be measured by Odin is given by Baron et al. [1] (this issue).

The closer an absorption band gets to meeting the following conditions the easier it will be to retrieve temperature and pressure [2].

(i) The emitting constituent should be uniformly mixed in the atmosphere so that the emitted radiance can be considered to be only a function of the temperature (at a given pressure level).

(ii) The absorption line or band should not overlap lines of other atmospheric constituents.

(iii) Local thermodynamic equilibrium (LTE) should apply so that the source function in a homogeneous layer at physical temperature $T$ is the Planck function corresponding to that temperature. As altitude increases, the collision rate decreases and at some altitude LTE will no longer be a good approximation.

(iv) The wavelength should be long enough so that the scattered solar radiation is insignificant compared with the thermal emission.

We also assume that hydrostatic equilibrium applies in the part of the atmosphere that we are interested in.

The two most-used well-mixed gases for $T / P$ retrievals are $\mathrm{CO}_{2}$ and $\mathrm{O}_{2}$. The first has been used for infrared measurements by, for example, LIMS (Limb Infrared Monitor of the Stratosphere) on the Nimbus-7 satellite [3], HALOE (Halogen Occultation Experiment) on board UARS (Upper Atmosphere Research Satellite) [4], but also by the close-to-nadir view system SSU (Stratospheric Sounding Unit) on the NOAA's (National Oceanographic and Atmospheric Administration) TIROS (Television Infrared Observation Satellite) series of satellites [5]. The use of the spin-rotation transitions of $\mathrm{O}_{2}$ for $T / P$ sounding was first suggested by Lenoir [6,7] and Gautier et al. [8]. $\mathrm{O}_{2}$ transitions have been used in the millimetre range for remote sensing of the atmospheric temperature since the NEMS (Nimbus E Microwave Spectrometer) instrument on the Nimbus-5 satellite [9]. Later, millimetre $\mathrm{O}_{2}$ transitions were observed in a limb-sounding view by MLS (Microwave Limb Sounder) on UARS [10] and MAS (Millimeter-wave Atmospheric Sounder) [11], and in a close-to-nadir view by MSU (Microwave Sounding Unit) (replaced by AMSU (Advanced Microwave Sounding Unit) in mid-1998) on TIROS-NOAA (see also ref. 5) and SSM/T1 (Special Sensor Microwave/Temperature) on the F-8 and successive DMSP 
(Defence Meteorological Satellite Program) satellites [12]. Sub-millimetre $\mathrm{O}_{2}$ lines have not been used on an operational basis for temperature and pressure retrievals so far, only some balloon-borne observations were reported by Pardo et al. [13].

The $\mathrm{O}_{2}$ line at $118.750 \mathrm{GHz}$ fulfills all the conditions stated above except the one concerning the overlapping. However, the choice of channels in Odin has been made so that this problem is minimized (discussed in Sect. 4.1). One peculiar aspect of atmospheric oxygen lines is that they show a Zeemansplitting effect. Since $\mathrm{O}_{2}$ has a permanent magnetic dipole it will interact with the Earth's geomagnetic field causing a splitting of the rotational energy levels. Below $\sim 60 \mathrm{~km}$ this effect becomes insignificant because the frequency splitting is negligible compared with the total width of the line and also because the high rate of collisions prevents the magnetic dipoles from effectively orienting along the magneticfield direction. However, at altitudes above $\sim 60 \mathrm{~km}$ it has been shown that this effect is important in accounting for ref. 13. The ability to accurately measure the temperature in the mesosphere is a crucial point for related mesospheric science to be performed. Gravity waves are the main issue. The importance of gravity waves for upper atmosphere structures has led to different experimental efforts including lidar, radar, and satellite optical observations. Odin will thus use a new technique in this field with the advantage of global coverage. These waves are generated at tropospheric altitudes because of effects such as the passage of fronts over intense pressure regions. The waves propagate upwards and grow in amplitude, until reaching a critical level near the mesopause, they "break" depositing energy and momentum [14]. The study of such phenomena is one of the scientific goals of the Odin temperature channels.

In this paper, we present (Sect. 2) the theory involved in the radiative transfer through $\mathrm{O}_{2}$ lines in the atmosphere and the forward models we developed (Sect. 3). Then we present the Odin $\mathrm{O}_{2}$ measurement modes (Sect. 4) and some simulations of atmospheric $\mathrm{O}_{2}$ spectra at $118.750 \mathrm{GHz}$ as viewed by the threechannel operational temperature sounder. We will estimate the importance of the Zeeman splitting for channel 1. Also in the same section, we present some estimations of high-resolution spectra at 118.75 and $487.25 \mathrm{GHz}$ that can be obtained by Odin using the $150 \mathrm{kHz}$ resolution acousto-optic spectrometer (AOS). These measurements are of great interest for checking the details of the radiative-transfer model we present below. We will also provide information about the strategy to be followed for temperature retrievals. Finally, our conclusions and a summary are given in Sect. 5. Simulations of the retrievals and evaluations of the expected accuracy is presented in a separate paper by Ridal et al. in this issue [15].

\section{Theory involved}

The radiation escaping from a homogeneous nonscattering layer $i$ in LTE in the atmosphere can be calculated, at a given frequency, according to

$I_{i}=I_{i-1} \mathrm{e}^{-g_{i} k_{i} l_{i}}+B_{i}\left(1-\mathrm{e}^{-g_{i} k_{i} l_{i}}\right)$

where $B$ is the blackbody function corresponding to the physical temperature of the layer, $l$ is the path length through the layer, $k$ is the absorption coefficient, and $g$ is the geometry factor $\mathrm{d} l / \mathrm{d} z$. In limbviewing geometry the factor $g$ can change dramatically from one layer to the next and is especially large in the tangent layer. To get around this problem the numerical integration has to be performed using variable step sizes and making a trade-off between speed and accuracy.

\subsection{Absorption coefficients}

The absorption coefficient at a given frequency $(v)$ in a nonscattering medium describes the fractional loss of intensity as radiation of this frequency passes through an homogeneous element of the medium. The emission coefficient $\epsilon_{v}$ expresses the emission of energy in the same element so that the change in the specific intensity of the radiation $\left(I_{v}\right)$ along a small path $\mathrm{d} s$ is

$$
\frac{\mathrm{d} I(v)}{\mathrm{d} s}=\epsilon_{v}-\kappa_{v} I(v)
$$


By Kirchoff's law, if LTE conditions are satisfied, the ratio between the absorption and emission coefficients equals the value of the blackbody spectrum at the physical temperature $T$ of the medium, $B_{v}(T)$, and this leads to the solution given in (1). The process of absorption between two molecular energy levels $u$ and $l$, takes place, in principle, only around the central frequency $v_{u \rightarrow l}$ with a distribution function known as the "natural" line profile, the halfwidth of which is given by Heisenberg's principle. In the atmosphere, however, the thermal motion of molecules and collisions between them modify this line shape to a much wider one. Combining microphysical knowledge of the process of line absorption or emission of energy by molecules with statistical studies of their behavior in systems containing a large number of them, we have the following general expression of the absorption coefficient (see, for example, refs. 2 and 16):

$\left(\kappa_{v}\right)_{l \rightarrow u}=\frac{8 \pi^{3} N v}{3 h c Q}\left[\mathrm{e}^{-E_{l} / k T}-\mathrm{e}^{-E_{u} / k T}<u|\boldsymbol{\mu}| l>^{2} f\left(v, v_{u \rightarrow l}\right)\right.$

where $N$ is the number density of molecules of the considered species in the considered vibrational state; $f\left(v, v_{u \rightarrow l}\right)$ is the line shape; $Q$ is the partition function, $Q=\sum_{i} g_{i} \exp \left(-E_{i} / k T\right) ; g_{i}$ is the statistical weight of the quantum state whose energy is $E_{i} ; \boldsymbol{\mu}$ is the dipole moment operator; and $\mid l>$ and $\mid u>$ are wave functions of the quantum states. Equation (3) is the basis for calculations of the absorption coefficient in the radiative transfer forward models we present below.

\subsection{Zeeman splitting}

$\mathrm{O}_{2}$ has a ${ }^{3} \Sigma_{\mathrm{g}}^{-}$ground electronic state. The $S=1$ permanent magnetic dipole comes from two parallel electron spins. Its value in debyes (equal to two Böhr magnetons) is 0.0186. The spin-rotation levels of this molecule are described by two quantum numbers: $N$ associated to the quantum operator $N$, which turns out to be the coupling of the electronic angular momentum operator $\boldsymbol{L}$ and the pure rotational angular momentum operator $\boldsymbol{O}$, and the total angular momentum quantum number $\boldsymbol{J}$ (the operator $\boldsymbol{J}$ is obtained by the coupling of $\boldsymbol{N}$ and the electronic spin $\boldsymbol{S}$ ). Owing to Bose-Einstein symmetry reasons, the quantum number $N$ has to be odd for ${ }^{16} \mathrm{O}_{2}$. The interaction of the magnetic dipole with the geomagnetic field leads to a Zeeman splitting of the ${ }^{16} \mathrm{O}_{2}(N, J)$ energy levels. If Hund's coupling case (b) were used to treat the sum of the three operators, $\boldsymbol{L}, \boldsymbol{O}$, and $\boldsymbol{S}$, the following expression of the splitting of $(N, J)$ levels would result:

$\Delta E=-1.0001 \mu_{0} M H \times \frac{J(J+1)+S(S+1)-N(N+1)}{J(J+1)}$

where $\mu_{0}$ is the Böhr magneton, $H$ is the magnetic field intensity, and $M$ is the magnetic quantum number $(M=-J, \ldots, 0, \ldots, J)$. However, Hund's ideal coupling-case (b) factor does not apply in all cases although it is very accurate for the $N_{J}=1_{1} \rightarrow 1_{0}$ transition at $118750.33 \mathrm{MHz}$, the one selected for Odin's operational temperature and pressure retrievals. This transition is also the simplest one from the point of view of Zeeman splitting; the lower energy level does not split and the upper level splits into three sublevels with magnetic quantum numbers $M_{U}=-1,0,+1$ (selection rules: $\Delta M=0, \pm 1$ ). Using (4) to obtain the frequency splitting in this particular transition, we have

$\Delta v(\mathrm{GHz})=-14.015 \times 10^{-6} H(\mu \mathrm{T}) M_{u}$

Applying the same equation to the the $487.25 \mathrm{GHz}$ transition, we have

$\Delta v(\mathrm{GHz})=-14.015 \times 10^{-6} H(\mu \mathrm{T})\left(\frac{1}{6} M_{u}-M_{l}\right)$

Another key point in regard with Zeeman splitting is its direction and polarization dependence. $\pi$ patterns $(\Delta M=0)$ arise with a linear polarization in the direction of the magnetic-field vector $(\boldsymbol{H})$. 
$\sigma \pm(\Delta M= \pm 1)$ radiation appears to be right-handed and left-handed circularly polarized in a plane perpendicular to $\boldsymbol{H}$. It turns out that depending on the line of sight and the orientation of the receiver's polarization, the observed line intensity and profile will change, and this is of the highest importance to retrieve other parameters from the observations, such as the temperature. To carry out the radiative transfer and take into account these phenomena, we have to incorporate full treatment of polarization.

One way is to formulate the radiative-transfer equation in terms of Stokes parameters or an equivalent notation such as the coherency matrix [17]. In fact, we have to fully propagate the electromagnetic wave field and then calculate the time average of the Poynting vector. To propagate the electric field along the $z$ direction we can write

$\boldsymbol{E}_{\boldsymbol{z}}=\exp (i \boldsymbol{k}[\boldsymbol{\Delta} z] n) \boldsymbol{E}_{\boldsymbol{o}} \equiv \exp \left(-R_{z} \Delta z\right) \boldsymbol{E}_{\boldsymbol{z}}$

where $n$ and $R_{z}$ are $2 \times 2$ complex matrices, the second one being called the refractivity matrix in the $x y$ or phase plane.

We will use, for our calculations, an auxiliary reference frame $(\boldsymbol{x}, \boldsymbol{y}, \boldsymbol{z})$ where $\boldsymbol{z}$ is the direction of propagation. The direction of the external magnetic field defines an angle $\phi$ with respect to $z$. For simplicity we will choose the direction of the $x$ axis so that $\boldsymbol{x}, \boldsymbol{z}$, and $\boldsymbol{H}$ are in the same plane (let us call $\boldsymbol{\tau}$ the unitary vector in the direction of $\boldsymbol{H}$ ). Another auxiliary reference frame will be defined by $\left(\boldsymbol{x}^{\prime}, \boldsymbol{y}, \boldsymbol{\tau}\right)$ where $\boldsymbol{x}^{\prime}=\boldsymbol{y} \times \boldsymbol{\tau}$. In this reference frame $\boldsymbol{x}^{\prime}$ and $\boldsymbol{y}$ define a plane perpendicular to the magnetic field. The refractivity matrix in the atmosphere near an $\mathrm{O}_{2}$ line affected by Zeeman splitting will be, in the $\left(\boldsymbol{x}^{\prime}, \boldsymbol{y}, \boldsymbol{\tau}\right)$ reference frame

$$
\begin{aligned}
R^{\prime}(\nu)=\left(\begin{array}{ccc}
n^{*}(v) & 0 & 0 \\
0 & n^{*}(v) & 0 \\
0 & 0 & n^{*}(\nu)
\end{array}\right)+S_{N_{J} \rightarrow N_{J^{\prime}}^{\prime}} \sum_{\pi} \zeta_{M} f_{M}(\nu)\left(\begin{array}{ccc}
0 & 0 & 0 \\
0 & 0 & 0 \\
0 & 0 & 1
\end{array}\right) \\
\\
+S_{N_{J} \rightarrow N_{J^{\prime}}^{\prime}} \sum_{\sigma+, \sigma-} \zeta_{M} f_{M}(\nu)\left(\begin{array}{lll}
1 & -i \delta_{ \pm} & 0 \\
i \delta_{ \pm} & 1 & 0 \\
0 & 0 & 0
\end{array}\right)
\end{aligned}
$$

where $n^{*}$ represents the isotropic contribution to the refractivity coming from the continuum and far wings of other lines that do not exhibit polarization, $S_{N_{J} \rightarrow N_{J^{\prime}}^{\prime}}$ is the rotational oscillator strength of the $\mathrm{O}_{2}$ line and $\zeta_{M}$ are the relative strengths of its Zeeman components, $f_{M}$ are individual line shapes for each individual component and $\delta_{ \pm}=1$ for $\sigma+$ lines and -1 for $\sigma-$ lines. The expressions to calculate the central positions and relative intensities of the Zeeman components for all the millimetre and submillimetre ${ }^{16} \mathrm{O}_{2},{ }^{16} \mathrm{O}_{2}(v=2)$, and ${ }^{16} \mathrm{O}^{18} \mathrm{O}$ lines have been reviewed by Pardo [18]. The coherency matrices accompanying the terms of the Zeeman components express their polarization characteristics (see above). Equation (8) assumes mutual incoherence between Zeeman components.

From the reference frame $\left(\boldsymbol{x}^{\prime}, \boldsymbol{y}, \boldsymbol{\tau}\right)$ to $(\boldsymbol{x}, \boldsymbol{y}, \boldsymbol{z})$ there is only a rotation by an angle $\phi$ around the $\boldsymbol{y}$ axis. The transformation of the refractivity matrix to the last frame is thus given by $R=L R^{\prime} L^{-1}$ where

$L=\left(\begin{array}{ccc}\cos \phi & 0 & -\sin \phi \\ 0 & 1 & 0 \\ \sin \phi & 0 & \cos \phi\end{array}\right)$

Of course, the phase plane of the wave is $x y$ since $z$ is the direction of propagation. The refractivity matrix restricted to the $x y$ plane will thus be

$R_{z}(v)=\left(\begin{array}{cc}n^{*}(v) & 0 \\ 0 & n^{*}(v)\end{array}\right)\left(\begin{array}{cc}R_{0} \sin ^{2} \phi+\left[R_{+}+R_{-}\right] \cos ^{2} \phi & -i\left[R_{+}-R_{-}\right] \cos \phi \\ i\left[R_{+}-R_{-}\right] \cos \phi & R_{+}+R_{-}\end{array}\right)$

where $R_{0}=S_{N_{J} \rightarrow N_{J^{\prime}}^{\prime}} \sum_{\pi} \zeta_{M} f_{M}(\nu)$ and $R_{ \pm}=S_{N_{J} \rightarrow N_{J^{\prime}}^{\prime}} \sum_{\sigma+, \sigma-} \zeta_{M} f_{M}(\nu)$. The $(\boldsymbol{x}, \boldsymbol{y})$ reference frame in the phase plane is not the final one that we will use in our problem since the orientation of 
Fig. 1. Reference frames used to compute the Zeeman effect on $\mathrm{O}_{2}$ molecules. $\boldsymbol{\tau}$ follows the direction of the magnetic field.

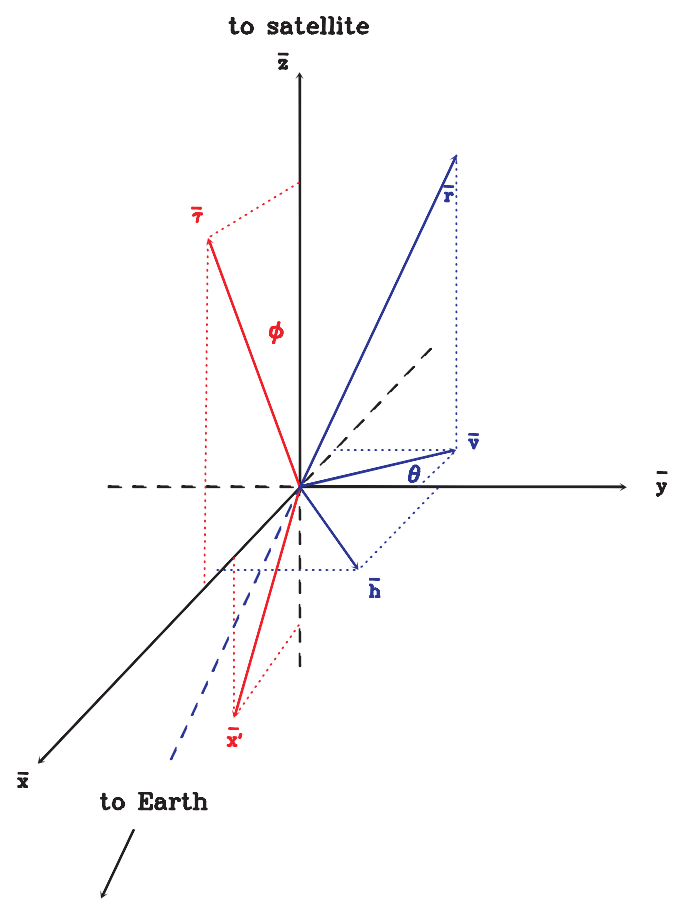

these two axes is determined by the direction of the local magnetic field and it changes along the path. The natural reference frame in the phase plane for the problem we are describing will be linked to the Earth, and is the one defined by the following vectors:

- $\boldsymbol{h}=\boldsymbol{r} \times \boldsymbol{z}(\boldsymbol{r}$ is the unitary vector along the geocentric direction $)$ that we define as the orientation of the linear horizontal polarization in the phase plane. Note that $r$ changes along the path. However, $\boldsymbol{r}$ and $z$ will always define the same plane since there is only one plane that contains $z$ and the center of the Earth for limb-sounding geometry. Thus, $\boldsymbol{h}$ defines the same direction at every point in the path.

- $\boldsymbol{v}=\boldsymbol{z} \times \boldsymbol{h}$ that we define as the direction of the linear vertical polarization.

From $(\boldsymbol{x} \boldsymbol{y})$ to $(\boldsymbol{h} \boldsymbol{v})$ there is only a rotation by an angle $\theta$ (that changes locally along the path). This angle can be calculated from $\tau, \boldsymbol{r}$ and $z$. All reference frames discussed in this section are illustrated in Fig. 1.

For observational purposes we are interested in calculating the brightness temperature, or equivalently, the energy flux arriving at the detector. For that we need to calculate the time average of the Poynting vector. Once such a calculation is performed for a homogeneous and isotropic layer beginning at $z_{\mathrm{i}}$ and ending at $z_{\mathrm{f}}$, using the fields resulting from (7), we arrive at a solution for the radiative-transfer equation (see details in ref. 13), which has the following form:

$I\left(z_{\mathrm{f}}, v\right)=\mathrm{e}^{-R_{z}(v) \Delta z} I\left(z_{\mathrm{i}}, v\right) \mathrm{e}^{-R_{z}^{* T}(v) \Delta z}+B(T, v)\left[1-\mathrm{e}^{-2 A_{z}(v) \Delta z}\right]$

where $I$ is a radiation intensity matrix in the $(2 \times 2)$ phase plane, the diagonal elements of which are associated with the intensity of the radiation in each polarization (two independent polarizations in the phase plane); $B(T, v)$ is the Planck function at frequency $v$ for a blackbody at physical temperature $T$; $A_{z}(v)=\left[R_{z}(v)+R_{z}^{* T}(v)\right] / 2$ is the Hermitian part of $R_{z}$; and $v$ is the frequency. 
Fig. 2. Ground-based measurements of the $2_{1} \rightarrow 0_{1}$ Zeeman split transition of ${ }^{16} \mathrm{O}^{18} \mathrm{O}$ from three different locations. Note the important changes in line profile. Slight asymmetries, specially notorious in the Mauna Kea spectrum, are due to the frequency-switch observation mode.

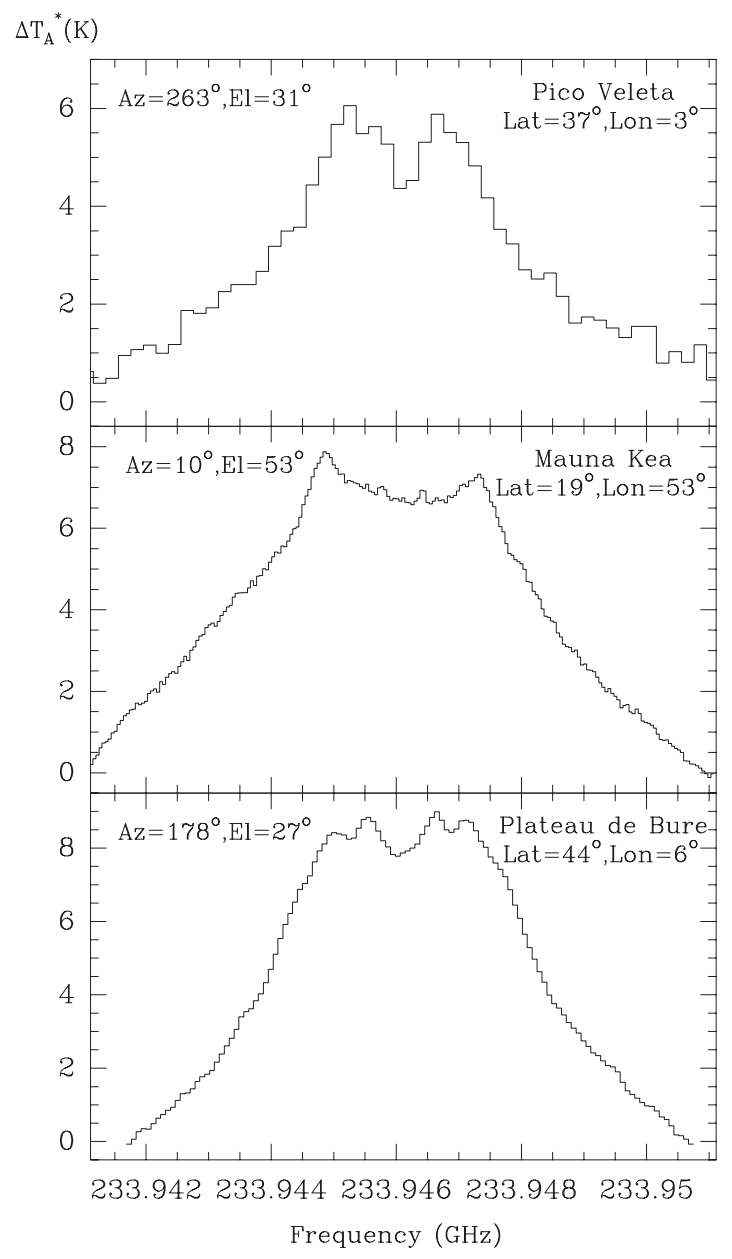

\subsection{Experimental evidence}

Several measurements have been reported that confirm the importance of the Zeeman splitting in the upper stratosphere and lower mesosphere. Hartmann et al. [19] measured the Zeeman structure of an $\mathrm{O}_{2}$ line within the $60 \mathrm{GHz}$ spin-rotation band at a tangent altitude of about $90 \mathrm{~km}$ using a receiver on board a NASA Space Shuttle; their instrument was used for temperature retrievals in the upper stratosphere and lower mesosphere [12]. Pardo et al. [13] and Sandor and Clancy [20] used an optically thin ${ }^{16} \mathrm{O}^{18} \mathrm{O}$ line at $233946.11 \mathrm{MHz}$ for ground-based observations of this effect. Some examples of different shapes that a spectrum of the latter line can have, as viewed from the ground at different locations and in different polarizations, are illustrated in Fig. 2.

\section{The forward models}

Simulations of $\mathrm{O}_{2}$ lines as viewed in limb-sounding geometry but without taking into account Zeeman splitting have been made with a forward model developed at the Meteorological Department of Stockholm University (MISU) together with the Department of Radio and Space Science at Chalmers 
University of Technology in Göteborg, and fully described by Eriksson et al. [21]. This forward model calculates the absorption coefficients for a number of layers, in a given frequency range. These coefficients are then used to solve the radiative-transfer equation for a number of steps through the line of sight. The atmosphere is divided into $0.5 \mathrm{~km}$ thick layers in which the temperature, pressure, and number density of the different atmospheric species are allowed to vary, by linear interpolation. The absorption coefficient of all the strongest lines (that will contribute a certain amount to the resulting radiation) of every atmospheric species considered are calculated for all frequencies and layers, according to (3) in the form

$\kappa_{v}=N S_{u \rightarrow l}(T) f\left(v, v_{u \rightarrow l}\right)$

where $N$ is the number density of the emitting/absorbing gas, $v_{u \rightarrow l}$ is the central frequency, $f\left(v, v_{u \rightarrow l}\right)$ is the line shape (currently the Voigt line shape [22] is used), and the parameter $S_{u \rightarrow l}(T)$ includes all the other parameters present in (3). The collisional half-widths and the $S$ parameters for all the lines of the molecules included are taken from a new line catalogue (Eriksson and Merino [23]), which is a compilation of the JPL catalogue [24] and the HITRAN database [25]. For molecular oxygen and water vapor, the spectroscopic parameters are taken from Liebe et al. [26].

The radiative-transfer model including Zeeman splitting, developed at the Observatoire de ParisMeudon (OPM), is fully described in Pardo et al. [18,27,28].

This model is based, for the lower part of the atmosphere (up to $\sim 60 \mathrm{~km}$ ), on (3) with transition probabilities calculated directly from the most recent Hamiltonians in the literature by Cernicharo [29] and line widths provided by Rosenkranz [30] and Liebe et al. [26]. The Zeeman effect on $\mathrm{O}_{2}$ lines in the mesosphere is included in this model as described in Sect. 2.2. For other molecules, (3) has been fully used and the transition probabilities have been also derived by Cernicharo from the most recent Hamiltonians in the literature. Collisional half-widths are taken from the HITRAN database.

\section{Odin T/P measurements}

In the spectral range of Odin, two $\mathrm{O}_{2}$ lines are accessible with the following central frequencies and quantum numbers: $118.75033 \mathrm{GHz}\left(1_{1} \rightarrow 1_{0}\right)$ and $487.24947 \mathrm{GHz}\left(3_{3} \rightarrow 1_{2}\right)$. Two different modes of $\mathrm{O}_{2}$ measurements are planned, a three-channel radiometer mode (the operational mode) and a high-resolution mode. The details and goals of these two modes are described below.

\section{1. $\mathrm{O}_{2}$ operational mode}

To avoid dedicating an entire high-resolution Odin/SMR spectrometer to the $T / P$ measurements, three fixed electronic filters (frequency bins) have been implemented and will be exclusively devoted to them. The position and width of these frequency bins have been selected to cover as large an altitude interval as possible. Bins with a bandwidth of $40 \mathrm{MHz}$ are relatively easy to produce. For Odin, the best frequency intervals were found to be

(1) $118.730-118.770 \mathrm{GHz} .20 \mathrm{MHz}$ on each side of the line center covers the highest altitudes $\sim 85 \mathrm{~km}$ and down to about 55-60 km. This channel will be sensitive to the Zeeman splitting.

(2) 118.800-118.840 GHz. This area in the "midwing" covers about $55 \mathrm{~km}$ down to $35-40 \mathrm{~km}$.

(3) 119.180-119.220 GHz. Rather far out in the wing. It covers $35 \mathrm{~km}$ down to about 25-30 km where the signal gets saturated and cannot be used.

Isotopic and vibrationally excited species of $\mathrm{O}_{2}$ should also be taken into account in the interval 118.0-119.5 GHz. Two lines are present: ${ }^{16} \mathrm{O}_{2}(v=2)$ at $119039.314 \mathrm{MHz}$ (well avoided by the bins) and ${ }^{16} \mathrm{O}^{18} \mathrm{O}$ at $118759.81 \mathrm{MHz}$. The second one falls into channel 1 of the radiometer and thus has to be taken into account when simulating the signal (see Fig. 3). Since we are confident in the value of the 
Fig. 3. Example of spectra with the frequency bins indicated. The line appearing in the left spectrum at about $119.04 \mathrm{GHz}$ belongs to vibrationally excited $\mathrm{O}_{3}$ and the other two $\left(118.36\right.$ and $119.28 \mathrm{GHz}$ ) belong to ${ }^{16} \mathrm{O}_{3}$.

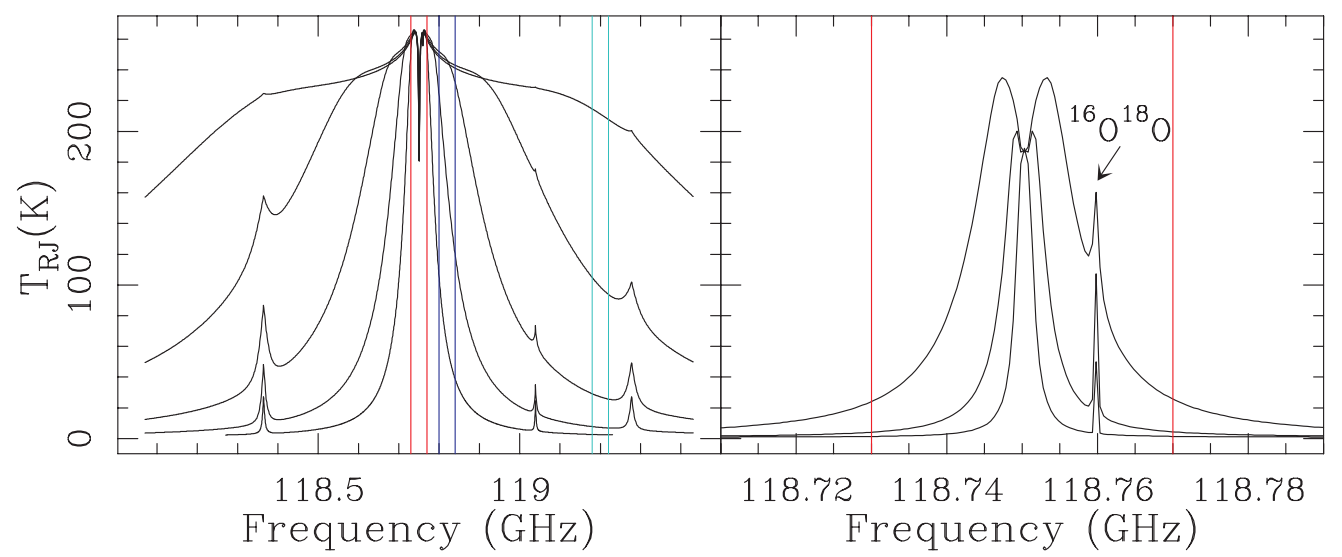

isotopic ratio, the presence of this line in channel 1 should not mean a major problem. The only weak point is the collisional width of this line, which has to be extrapolated from the value of the $118.75 \mathrm{GHz}$ ${ }^{16} \mathrm{O}_{2}$ line.

Figure 3 shows ${ }^{16} \mathrm{O}_{2}$ emission spectra for several limb paths with the frequency bins indicated. The signal within these bins as a function of altitude is shown in Fig. 4. This figure illustrates how the three altitude domains in which the signal is optically thin for the three channels cover the region 25 to $90 \mathrm{~km}$ in a quite optimized way. These altitude intervals may slightly vary depending on the atmospheric conditions as shown in the same figure.

The frequency choice has also been determined by taking into account the presence of lines from non- $\mathrm{O}_{2}$ important species, such as $\mathrm{H}_{2} \mathrm{O}$ and $\mathrm{O}_{3}$. We have analyzed this effect for the vertical domain to be covered $(\sim 25-95 \mathrm{~km})$ and found a negligible contribution to the opacity due to water vapor but the presence of two relatively important ozone lines with the following central frequencies: $118.36451 \mathrm{GHz}$ and $119.27765 \mathrm{GHz}$. At a tangent altitude of $30 \mathrm{~km}$ these lines show along the path a peak opacity of 0.35 and 0.14 , respectively, whereas the $\mathrm{O}_{2}$ opacity at the central position of these $\mathrm{O}_{3}$ lines is 0.85 and 0.47 . However, the position of the three channels of the Odin $T / P$ operational system were chosen to avoid these two lines. They are almost negligible for the three channels over the entire vertical range. As for the $\mathrm{O}_{2}$ line at $487.25 \mathrm{GHz}$, it might be contaminated by another $\mathrm{O}_{3}$ line at $487.34851 \mathrm{GHz}$, which reaches a peak opacity of 20 for the same path (30 km tangent point). However, the lower frequency wing of the $\mathrm{O}_{2}$ line stays free from overlapping lines for at least $1 \mathrm{GHz}$ from the line center.

\subsection{High resolution $\mathrm{O}_{2}$ spectroscopy}

It has been proposed to use the AOS spectrometers in the highest spectral resolution modes $(\delta v=$ $150 \mathrm{kHz}$ ) for a few occasions to fine-measure the structure of the two $\mathrm{O}_{2}$ lines reachable by Odin. The main interest is to carefully validate the radiative-transfer model that will be used to analyze the data obtained in the operational three-channel mode. Also, the high-resolution data will provide information about the strength of the geomagnetic field in some selected locations to check the validity of the standard IGRF model (ref. 31, see below) used also in our retrieval code. A maximum frequency resolution of $150 \mathrm{kHz}$ will allow a maximum sensitivity to the magnetic-field strength of $10.70 \mu \mathrm{T}$ by measuring the two external $\sigma \pm$ components of the $118.75 \mathrm{GHz}$ line, and $6.42 \mu \mathrm{T}$ by measuring the $\pi(M=+2 \rightarrow+2, M=-2 \rightarrow-2)$ components of the $487.25 \mathrm{GHz}$ line. The expected extreme values of the geomagnetic field are 22 and $65 \mu \mathrm{T}$. 
Fig. 4. Left: Example of height profiles corresponding to the three frequency bins. Continuous line: tropical atmosphere; broken line: midlatitude winter atmosphere; dotted line: subpolar winter atmosphere (from the U.S. Standard 1976 atmospheres [32]). Right: Differences between LH and LV brightness temperatures expected for channel $1( \pm 20 \mathrm{MHz}$ around line center) divided by the brightness temperature corresponding to the case with no magnetic field. Each curve corresponds to a different latitude marked on the figure.
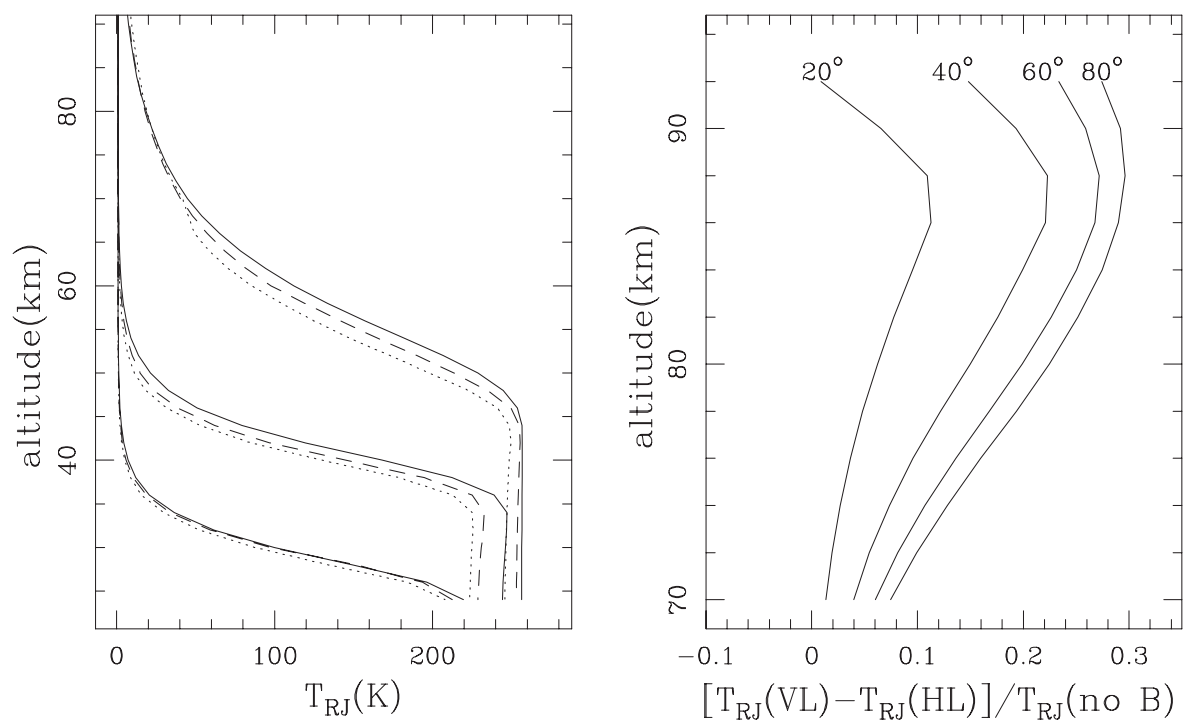

Fig. 5. Simulated high-resolution $\mathrm{O}_{2}$ spectra at $92 \mathrm{~km}$ tangent point when the satellite is above the Greenwich meridian at $60^{\circ}$ latitude looking South. Continuous line: HL polarization; broken line: VL polarization. Left: 118.75 GHz line. Right: $487.25 \mathrm{GHz}$ line.
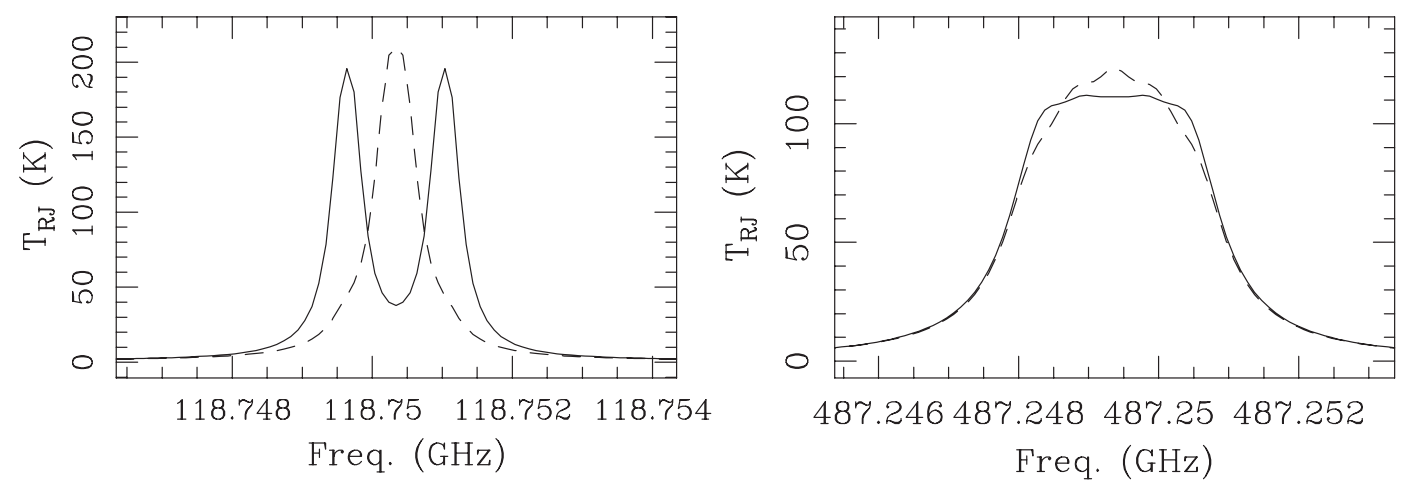

In Fig. 5 we show some simulations of $\mathrm{O}_{2}$ spectra for the maximum-resolution mode expected using the autocorrelators on board Odin. The atmospheric $T / P$ vertical profile was taken from the U.S. Standard 1976 Atmosphere [32] for the latitude range concerned. The calculations of the geometric path include a flatness factor of $1 / 298.2$ for the Earth. The geomagnetic-field vector is obtained from the International geomagnetic reference field [31], the empirical representation of the Earth's magnetic field recommended by the International Association of Geomagnetism and Aeronomy (IAGA). This model employs a spherical harmonics expansion of the scalar potential in geocentric coordinates. The set of expansion coefficients corresponding to 1985 has been selected for these simulations. 


\subsection{Retrieval strategy}

A retrieval procedure has been specifically developed at MISU to be applied to the filter-bank three-channel operational mode with inputs from the OPM model for the Zeeman-affected channel 1. The retrievals are performed using the iterative feedback method in which the simulated signal from a reference atmosphere with known temperature and pressure is used as a lookup table to obtain the pressure at the measurement points. A temperature profile is calculated using the equation of hydrostatic equilibrium. From this new reference atmosphere a new lookup table can be derived. The solution is iterated, using the retrieved temperature/pressure profile as the new reference, until the difference between two iterations is less than a given limit of convergence. The methodology is fully described by Baron et al. [1].

\section{Conclusions}

The Odin satellite is the first satellite to retrieve temperature and pressure in the upper stratosphere and mesosphere by using the limb-sounding technique to monitor the $118.75 \mathrm{GHz}{ }^{16} \mathrm{O}_{2}$ resonance. The pressure data are specially important to establish a vertical grid to which data of other atmospheric molecules measured by Odin will be referred. The temperature information is interesting for radiative balance studies in the upper stratosphere and mesosphere; for research on atmospheric gravity waves; or, as shown in this paper, for geomagnetic-field studies.

The ${ }^{16} \mathrm{O}_{2}$ measurements at $487 \mathrm{GHz}$ will be the first satellite-borne sub-millimetre observations of $\mathrm{O}_{2}$ and, though experimental in Odin, will provide data with a much better vertical resolution at the tangent altitude than any previous measurement.

\section{Acknowledgements}

J.R. Pardo gratefully acknowledges the financial support of the Centre National d'Études Spatiales (France) and Météo-France for the development of this work. He also acknowledges Observatoire de Grenoble, Institut de Radioastronomie Millimétrique (IRAM), and CalTech Submillimetre Observatory (NSF grant AST-9980846) for the use of their facilities for some measurements presented in this paper. The work by the MISU group was supported by grants from the Swedish National Space Board.

\section{References}

1. P. Baron, P. Ricaud, J. de la Noë, J.E.P. Eriksson, F. Merino, and D.P. Murtagh. Can. J. Phys. 80 (2002). This issue.

2. J.T. Houghton, F.W. Taylor, and C.D. Rodgers. Remote soundings of atmospheres. Cambridge University Press, Cambridge. 1984.

3. J.C. Gille, J.M. Russel, P.L. Bailey, L.L. Gordely, E.E. Remsberg, J.H. Lienesch, W.G. Planet, F.B. House, L.V. Lyjak, and S.A. Beck. J. Geophys. Res. 89, 5147 (1984).

4. M.E. Hervig, J.M. Russel, L.L. Gordely et al. J. Geophys. Res. 101, 10277 (1996).

5. W.L. Smith, H.M. Woolf, C.M. Hayden, D.Q. Wark, L.M. Mcmillin. Bull. Am. Meteorol. Soc. 60, 1177 (1979).

6. W.B. Lenoir. J. App. Phys. 38(13), 5283 (1967).

7. W.B. Lenoir. J. Geophys. Res. 73, 361 (1968).

8. D. Gautier and A. Robert. Ann. Geophys. 20, 480 (1964).

9. J.W. Waters, K.F. Kunzi, R.L. Pettyjohn, R.K.L. Poon, and D.H. Staelin. J. Atmos. Sci. 32(10), 1953 (1975).

10. E.F. Fishbein, R.E. Cofield, L. Froidevaux. J. Geophys. Res. 101, 9983 (1996).

11. A. von Engeln, J. Langen, T. Wehr. J. Geophys. Res. 103(D24), 31735 (1998).

12. H.J. Kramer. Observation of the Earth and its environment - Survey of missions and sensors. 2nd ed. Springer-Verlag, Berlin. ISBN: 3-540-578587. 1994.

13. J.R. Pardo, L. Pagani, G. Olofsson, P. Febvre, and J. Tauber. J. Quantum. Spectrosc. Radiat. Transfer, 67(2), 169 (2000). 
14. C.Y. She, J.R. Yu, D.A. Krueger, R. Roble, P. Keckhut, A. Hauchecorne, and M.-L. Chanin. Geophys. Res. Lett. 22, 377 (1995).

15. M. Ridal, D.P. Murtagh, F. Merino, J.R. Pardo, and L. Pagani. Can. J. Phys. 80 (2002). This issue.

16. H.W. Kroto. Molecular rotation spectra. John Wiley \& Sons Ltd., New York. 1975. p. 67.

17. M. Born and E. Wolf. Principles of optics. 6th ed. Pergamon Press, Oxford. 1984. pp. 544-550.

18. J.R. Pardo. Thèse de doctorat, Universite de Paris VI. 1996.

19. G.K. Hartmann, W. Degenhardt, M.L. Richards et al. Geophys. Res. Lett. 23, 2329 (1996).

20. B.J. Sandor and R.T. Clancy. Geophys. Res. Lett. 24(13), 1631 (1997).

21. J.E.P. Eriksson, F. Merino, D. Murtagh, P. Baron, P. Ricaud, and J. de la Noë. Can. J. Phys. 80 (2002). This issue.

22. S.R. Drayson. J. Quantum. Spectrosc. Radiat. Transfer, 16, 611 (1976).

23. J.E.P. Eriksson and F. Merino. Research Rep. No. 179, Chalmers University of Technology, Göteborg, Sweden. 1997.

24. H.M. Poynter and D.E. Picket. Appl. Opt. 24(14), 2235 (1985).

25. L.S. Rothman, R.R. Gamache, R.H. Tipping et al. J. Quantum. Spectrosc. 48, 469 (1992).

26. H.J. Liebe, G.A. Hufford, and M.G. Cotton. Proceedings of the NATO/AGARD Propagation Panel. 52nd Specialists' Meeting of the Electromagnetic Wave Propagation Panel. 17-20 May 1993, Palma de Mallorca, Spain. No. 3, 1-10.

27. J.R. Pardo, L. Pagani, M. Gerin, and C. Prigent. J. Quantum Spectrosc. Radiat. Transfer, 54, 931 (1995).

28. J.R. Pardo, J. Cernicharo, and E. Serabyn. IEEE Trans. Antennas Propag. 49(12), 1683 (2001).

29. J. Cernicharo. Thèse de doctorat d'Etat, Université de Paris VI. 1988.

30. P.W. Rosenkranz. J. Quantum. Specrosct. Radiat. Transfer, 39(4), 287 (1988).

31. D.R. Barraclough. Phys. Earth Planet. Inter. 48, 279 (1987).

32. U.S. Commitee on Extension to the Standard Atmosphere. U.S. Printing Office, Washington, D.C. 1976. 